ARTICLE

\title{
Multi-Group Constants Generation System for 3D-Core Simulation Using a Continuous Energy Monte Carlo Technique
}

\author{
Kenichi YOSHIOKA ${ }^{1, *}$, Yutaka TAKEUCHI ${ }^{2}$, Taku KITAMURA² and Shungo SAKURAI ${ }^{2}$ \\ ${ }^{1}$ TOSHIBA Corporation, Power Systems Company, Kawasaki, Japan \\ ${ }^{2}$ TOSHIBA Corporation, Power Systems Company, Yokohama, Japan
}

\begin{abstract}
A Monte Carlo method is a powerful and flexible approach for calculating heterogeneous fuel designs with the aim of developing innovative fuel and core concepts, as the calculation precisely handles both the geometrical configurations of a fuel assembly and the contained materials therein. However, a whole-core Monte Carlo burn up calculation has not been feasible, because it requires large scale computing resources and time. Instead, a combination of a Monte Carlo method and a conventional deterministic method is more applicable. The continuous energy Monte Carlo technique is used to generate the multi-group constants of a fuel assembly for a three-dimensional core simulation, which uses a conventional deterministic method.

We have developed a multi-group constants generation system for a three-dimensional core simulator using the results of a continuous energy Monte Carlo simulation with reasonable computation time. We also performed a transient calculation based on the three-dimensional core simulation made by the system.

We have performed verification analyses of the system using current BWR fuels and a BWR core configuration. Compared with the current method, namely a multi-group constants generation system using the results of a deterministic lattice physics code, the result of the newly developed system has shown good compatibility with the current system on both core performance simulations and plant transient simulations.
\end{abstract}

KEYWORDS: continuous energy Monte Carlo, deterministic method, multi-group constants generation, 3-D core simulation, transient calculation

\section{Introduction}

We need to develop a fuel and core analysis system in order to efficiently and precisely develop next generation nuclear fuels and cores with, for example, an over-5\% enriched fuel, a super high burnup fuel, a mixed-oxide fuel core, a long life cycle core, or a high power density core. We have been developing a next generation fuel and core analysis system based on the continuous energy Monte Carlo technique which can precisely handle both the complex geometrical configurations and the material heterogeneities of the aforementioned fuels and cores.

The continuous energy Monte Carlo technique can simulate various, complex geometrical configurations, can precisely treat a self-shielding effect, and is one of the best methods for fuel design and analyses with complicated geometries and enrichment distributions. With the latest development in computer performance, the continuous energy Monte Carlo burnup technique has become commonly applied ${ }^{1,2)}$ while the whole-core Monte Carlo technique has been applied in the case of an initial core calculation. However, a whole-core Monte Carlo burnup technique with thermal-hydraulic feedbacks still requires a large amount of computing resources and time. Therefore, a combination of a Monte Carlo technique and a conventional deterministic method is both attractive and feasible. A continuous energy

*Corresponding author,E-mail:kenichi.yoshioka@toshiba.co.jp

(C) 2011 Atomic Energy Society of Japan, All Rights Reserved
Monte Carlo technique is used to generate the multi-group constants of a fuel assembly. Then, a nuclear-thermo coupled 3-D core simulation is performed using a conventional deterministic method. One proposition was to use a cross-section generation method for a BWR core calculation with the continuous-energy Monte Carlo technique. ${ }^{3)}$ However, a method that precisely evaluates a scattering matrix, including self-scattering and up-scattering cross-sections has not yet been established in Ref. 3). In order to cope with this situation, one of the authors has proposed a method featuring a multi-group scattering matrix generation via a weight-to-flux ratio, and developed a multi-group constants generation system by this method. ${ }^{4)}$

We have developed a multi-group constants generation system for a BWR 3-D core simulator based on a three neutron group nodal expansion method by extending the above multi-group constants generation system. The system is able to evaluate discontinuity factors in addition to cross-sections and diffusion coefficients.

This paper presents the developed multi-group constants generation system for a BWR 3-D core simulator, the application to a BWR 3-D core calculation and the plant transient analysis based on the calculated core.

Section II compares the multi-group constants generated by the developed system to a current design system for a BWR high burnup fuel; Section III describes major core performances of ABWR equilibrium core by the developed system; Section IV shows the application results for ABWR 
plant transient analysis; and Section V gives a brief conclusion.

\section{Multi-Group Constants Generation for 3-D Core Simulator}

\section{Multi-Group Constants Generation by Continuous Energy Monte Carlo Technique}

In this section, a multi-group constants generation system for a BWR 3-D core simulator is shown.

The objective core simulator, NEREUS, ${ }^{5)}$ is based on the three neutron group diffusion theory modeled with a nodal expansion method. In a current design procedure, NEREUS uses three group constants generated by a deterministic fuel assembly design code TGBLA. ${ }^{6)}$ We have developed a multi-group constants generation system by a continuous energy Monte Carlo burn up calculation code, MCNP-BURN2, ${ }^{1)}$ instead of TGBLA.

Historical and instantaneous parameter dependent multi-group constants are required for NEREUS calculation such as diffusion coefficient, production cross-section, absorption cross-section by poison nucleus, neutron detector fission cross-section, discontinuity factor and so on. These multi-group constants are generated using various reaction rates and neutron flux which are calculated utilizing MCNP-BURN2 in accordance with the definition of each parameter. The diffusion coefficient is calculated with the generated scattering cross-section and the mean scattering cosine of the Monte Carlo calculation. The discontinuity factor is calculated with the fuel assembly average neutron flux, assembly four side surface neutron flux and assembly four side corner neutron flux.

In order to include the gamma heating into the power distribution, neutron and photon coupled Monte Carlo simulation is adopted.

The method for generating multi-group constants is described using the MCNP code. ${ }^{7)}$

If we assume that volume $\mathrm{V}$ consists of a homogeneous material, then neutron flux is expressed using the track length estimator of the MCNP code as follows.

$$
\phi=\sum_{i} \frac{w_{i} \cdot T l_{i}}{V},
$$

where

$w_{i}$ : the i-th weight,

$T l_{i}$ : the i-th track length,

$V$ : volume,

$\Sigma_{i}$ : the sum of the track length passing through $\mathrm{V}$.

After obtaining neutron flux $\phi$, the reaction rates are expressed as follows.

\section{Absorption reaction rate: $\Sigma_{\mathrm{a}} \times \phi$}

Production reaction rate: $v \Sigma_{\mathrm{f}} \times \phi$

Fission reaction rate: $\Sigma_{\mathrm{f}} \times \phi$

Therefore, the $g$ th energy group absorption cross-section can be written as,

$$
\Sigma_{a g}=\frac{\int_{g} \Sigma_{a}(E) \phi(E) d E}{\int_{g} \phi(E) d E}
$$

where the above cross-section means the cross-section averaged over volume $V$. Both the numerator and denominator of Eq. (2) are obtained from the results of the MCNP code. In this way, the absorption cross-section and the production cross-section are easily processed in the continuous energy Monte Carlo calculation. On the other hand, it is not possible to process the scattering cross-section from energy group $g$ to energy group $g$ ' in the same way. The energy's starting point and arrival point are needed to evaluate the scattering cross-section. These values are usually not satisfied by a continuous energy Monte Carlo code such as MCNP.

Moreover, the transport cross-section, which is utilized to obtain the diffusion coefficient $D$, is not easily processed as it requires the mean scattering cosine $\mu$, the scattering cross-section $\Sigma_{\mathrm{s}}$ and the absorption cross-section $\Sigma_{\mathrm{a}}$ as,

$$
\Sigma_{\mathrm{tr}}=\Sigma_{\mathrm{a}}+(1-\mu) \Sigma_{\mathrm{s}} \quad\left(=\Sigma_{\mathrm{t}}-\mu \Sigma_{\mathrm{s}}\right) .
$$

The diffusion coefficient $D$ is defined with the transport cross-section as

$$
D=\frac{1}{3 \Sigma_{t r}}
$$

where anisotropic diffusion is not considered.

The scattering cross-section is evaluated by using weight-to-flux ratio method. We describe briefly the method by using 3 energy groups as an example. We classify weight $w$ according to in-scattering group and out-scattering group.

Taking energy group number 3 , which consists of thermal neutrons, as an example, we can write as follows.

$$
w_{3}=w_{3}(1 \rightarrow 3)+w_{3}(2 \rightarrow 3)+w_{3}(3 \rightarrow 3)+w_{3}(0),
$$

where

$w_{3}(1 \rightarrow 3)$ : the weight generated through scattering in the energy group 1 ,

$w_{3}(2 \rightarrow 3)$ : the weight generated through scattering in the energy group 2 ,

$w_{3}(3 \rightarrow 3)$ : the weight generated through self-scattering in the energy group 3 ,

$w_{3}(0)$ : the weight generated directly by a fission source or the weight coming from a neighboring region.

The classified weights are then tallied. In Eq. (5), we omit $w_{3}(0)$ in the fission term, which is normally negligible, because almost all neutrons emitted from fission reactions are fast neutrons.

A scattering matrix is then written as the weight-to-flux ratio.

$$
\begin{aligned}
& \Sigma_{s 1 \rightarrow 3}=\frac{w_{3}(1 \rightarrow 3)}{w_{1} T l_{1}}=\frac{w_{3}(1 \rightarrow 3)}{\phi_{1}} \\
& \Sigma_{s 2 \rightarrow 3}=\frac{w_{3}(2 \rightarrow 3)}{w_{2} T l_{2}}=\frac{w_{3}(2 \rightarrow 3)}{\phi_{2}}, \\
& \Sigma_{s 3 \rightarrow 3}=\frac{w_{3}(3 \rightarrow 3)}{w_{3} T l_{3}}=\frac{w_{3}(3 \rightarrow 3)}{\phi_{3}}
\end{aligned}
$$

In the MCNP calculation, the scattering matrix can be obtained by these equations.

A weight is tallied according to the in-scattering group and the out-scattering group when the weight experiences a scattering reaction in volume $V$. Multi-group constants such as diffusion coefficients and reaction cross-sections includ- 


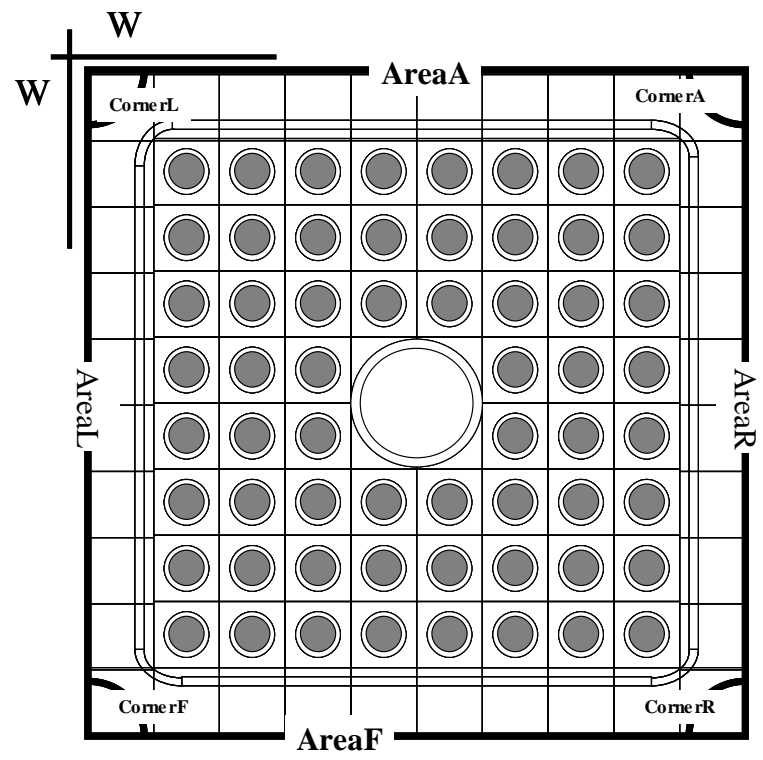

Fig. 1 Surface for discontinuity factor calculation

ing the scattering matrix are prepared according to the procedure. The mean scattering cosine $\bar{\mu}$ is needed to deduce the diffusion coefficients. Then, $\mu$ is tallied when the neutrons experience scattering. By averaging these tallied $\mu$ values, $\bar{\mu}$ is calculated. The diffusion coefficient $D$ is consequently deduced through Eq. (4).

Discontinuity factors of the boundaries of an assembly were defined as follows. The surfaces for the discontinuity factors were shown by AreaA, AreaL, AreaR and AreaF in Fig. 1. The range of integration in numerator is over a surface, while the range of integration in denominator is over a whole volume.

$$
\begin{aligned}
& A D F A g=\frac{\int_{r \in \text { AreaA }} d r d \Omega \int_{E_{g-1}}^{E_{g}} d E \phi(r, E, \Omega)}{\int_{r \in \text { AreaA }} d r} / \frac{\int_{r \in \text { WholeVolune }} d r d \Omega \int_{E_{g-1}}^{E_{g}} d E \phi(r, E, \Omega)}{\int_{r \in \text { WholeVolune }} d r}
\end{aligned}
$$

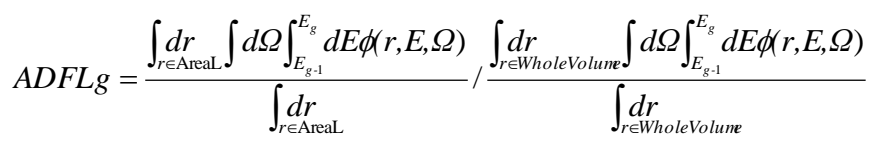

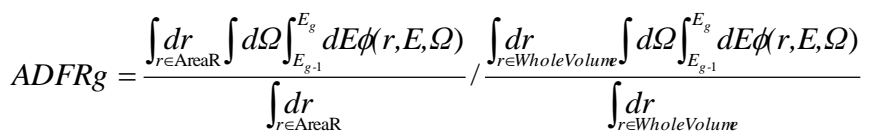

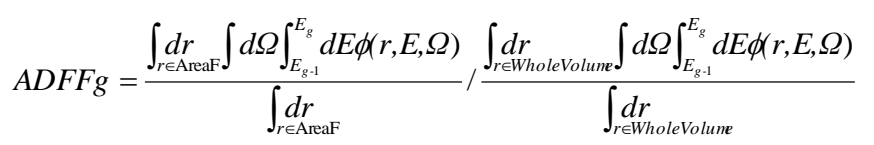

Discontinuity factors of the corners of the assembly were defined as follows. An annular cell was assumed at each corner. The scalar flux of the surface of the annular cell was used for the calculation of the discontinuity factor. The corners for the discontinuity factors were shown by CornerL, CornerR, CornerA, CornerF in Fig. 1. The range of integration in numerator is over a surface, while the range of

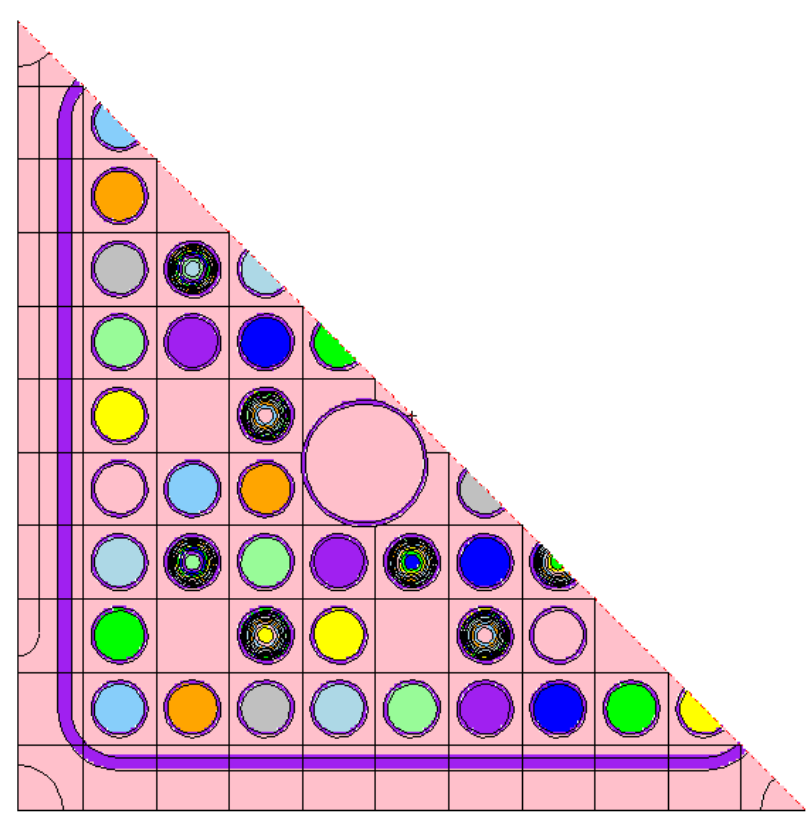

Fig. 2 Calculation geometry of fuel assembly

integration in denominator is over a whole volume.

$C D F L g=\frac{\int_{r \in \text { Comerl }} d r}{\int d \Omega \int_{E_{g-1}}^{E_{g}} d E \phi(r, E, \Omega)} / \frac{\int_{r \in \text { WholeVolune }} d d \Omega \int_{E_{g-1}}^{E_{g}} d E \phi(r, E, \Omega)}{\int d r}$

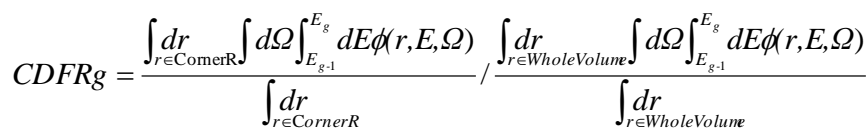

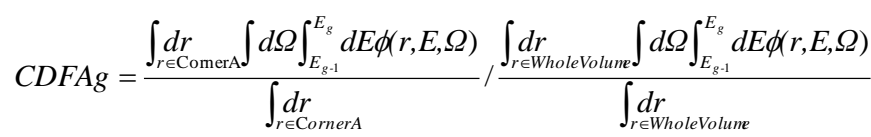

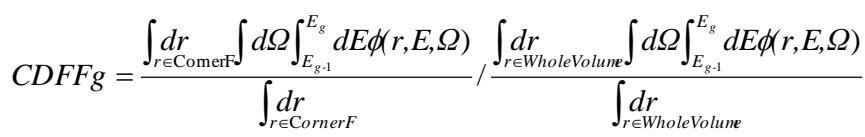

\section{Comparison with Deterministic Method}

We have compared the developed system based on the MCNP-BURN2 calculations with the current system based on the TGBLA calculations on the multi-group cross-sections, k-infinity and the discontinuity factor for the STEP-IIIA type fuel installed in an ABWR core. We used JENDL3.3 $3^{9)}$ as the nuclear data library for the MCNP-BURN2 calculation. The TGBLA calculations were based on ENDF-B/V. We used the half symmetry analytical configuration as shown in Fig. 2, considering the fuel assembly symmetry.

$\mathrm{UO}_{2}$ rods were treated as one region. Gadolinia bearing $\mathrm{UO}_{2}$ rods were divided into 10 regions in the radial direction. Water rods and channel box were assumed as same as the real dimension. Reflective boundary condition was used for the axial boundary. Table 1 shows the summary of the 
Table 1 Analytical condition for MCNP-BURN2

\begin{tabular}{cl}
\hline Exposure Step & 0.00 .20 .51 .02 .03 .04 .05 .06 .07 .08 .09 .010 .0 \\
{$[\mathrm{GWd} / \mathrm{t}]$} & 11.012 .013 .014 .015 .017 .520 .025 .030 .035 .0 \\
& 40.045 .050 .055 .060 .065 .070 .075 .0
\end{tabular}

\begin{tabular}{|c|c|}
\hline Power Density & $50.5 \mathrm{~W} / \mathrm{cc}$ \\
\hline Fuel Type & ABWR STEP-IIIA \\
\hline Temperature & Fuel $900 \mathrm{~K} \quad$ Clad $600 \mathrm{~K} \quad$ Moderator $600 \mathrm{~K}$ \\
\hline Library & JENDL-3.3 \\
\hline \multirow{2}{*}{ History } & Burnup $10,000 \times 50$ cycle \\
\hline & Group Constants Generation $10,000 \times 100$ cyc \\
\hline
\end{tabular}

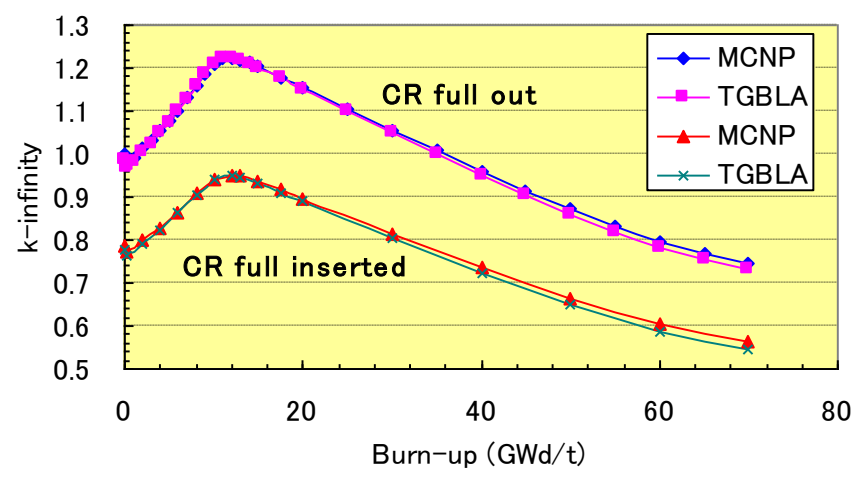

Fig. 3 Comparison of k-infinity between MCNP and TGBLA

analytical condition. In the first step, isotope compositions in each region are calculated through the burnup calculation with MCNP-BURN2. Then, multi-group constants are calculated using the isotope composition. In the second step, void reactivity, Doppler reactivity, control rod reactivity and so on are calculated. To keep high precision of those reactivities, more histories for the second step are needed than that for the first step.

Figure 3 shows the comparisons of k-infinity between the developed system and the current system. The control rod full drawn case and control rod full inserted case were calculated at the $40 \%$ void condition, respectively. The maximum difference was about $1.7 \% \mathrm{dk}$ at the end of cycle and the average difference was less than $0.8 \% \mathrm{dk}$, which is relatively small, taking into account the library difference. ${ }^{8,9)}$

Figure 4 shows the comparisons of slowing down cross-sections. The title of abscissa, neutron group number 1 means the down scattering from group 1 to 2 , and 2 means 2 to 3 .

In this figure, the cross-sections at the cycle exposure of $0 \mathrm{GWd} / \mathrm{t}$, and void fraction of $0 \%, 40 \%$ and $70 \%$ were compared. The difference was less than $2.0 \%$, so it was found that the scattering cross-sections are evaluated properly with the developed system.

Figure 5 shows the comparison of discontinuity factors of 4 surface sides of the assembly and 4 corner sides of the assembly, respectively. The factors were calculated at $0 \mathrm{GWd} / \mathrm{t}$ and $40 \%$ void condition, in this example. The number fol-

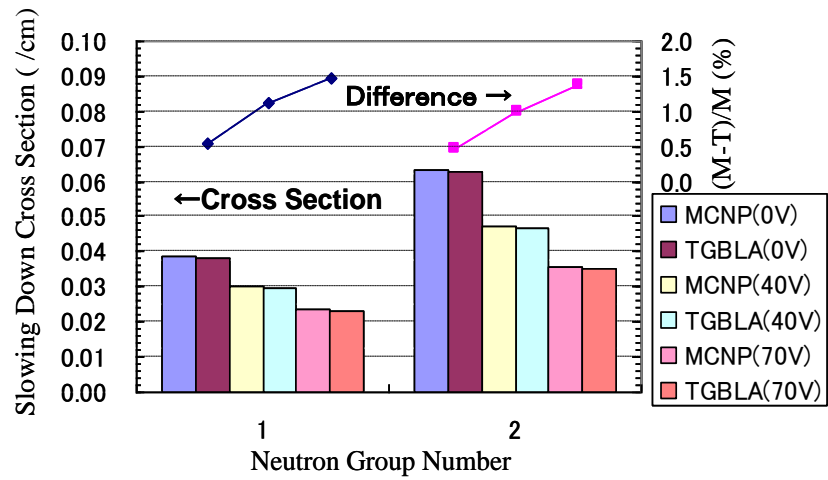

Fig. 4 Comparison of down scattering cross-sections

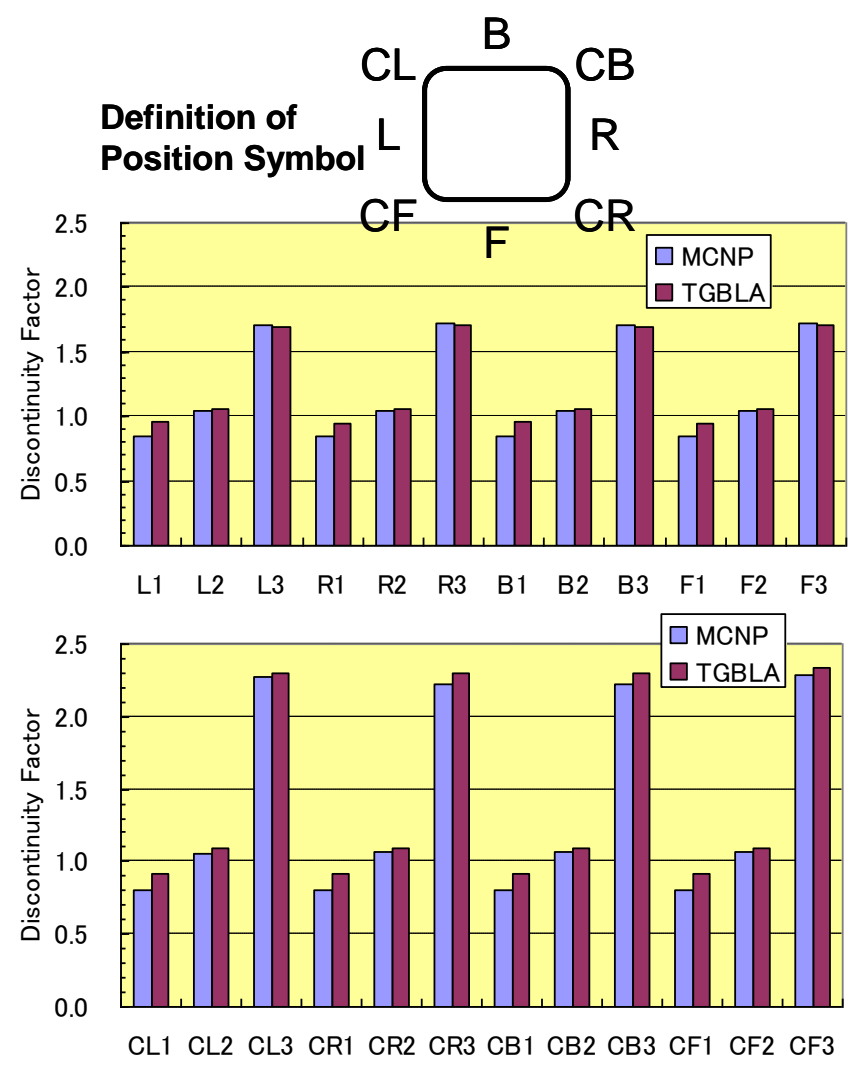

Fig. 5 Comparison of discontinuity factors

lowing the position symbol means the neutron group number. The differences of group 2 and 3 were less than $3 \%$. Those of the side surface were less than $1 \%$. The difference of group 1 by more than $10 \%$ is relatively large. TGBLA uses the diffusion theory to calculate neutron flux in the assembly while MCNP uses the transport theory. These theories might cause the difference of group 1.

By comparison, the multi-group constants generation using a continuous Monte Carlo technique is a proper method, and consistent with the deterministic method.

\section{Application to Core Performance Analysis}

Using the three group constants generated with MCNP-BURN2 and TGBLA as mentioned in Section II, we made the equilibrium ABWR core after 13 months of 
Table 2 Analytical condition for core design

\begin{tabular}{ll}
\hline Core thermal power (MW) & 3926 \\
\hline Rated core flow $(\mathrm{t} / \mathrm{h})$ & 52219 \\
\hline Core flow window & $90-110 \% /$ rated \\
\hline Number of fuel assemblys & 872 \\
\hline MLHGR $(\mathrm{kW} / \mathrm{m})$ & $\leqq 44$ \\
\hline MCPR & $\geqq 1.35$ \\
\hline Operation cycle & $13 \mathrm{months}$ \\
\hline Average discharge burnup & $45 \mathrm{GWd} / \mathrm{t}$ \\
\hline
\end{tabular}

\begin{tabular}{|c|c|c|c|c|c|c|c|c|c|c|c|c|c|c|c|}
\hline & & & & & & & & & & & & & & & \\
\hline & & & & & & & & & & & & & 2 & 4 & \\
\hline & & & & & & & & & & 1 & 4 & 5 & 4 & & \\
\hline & & & & & & & 1 & & & 4 & & 4 & 2 & 5 & \\
\hline & & & & & & 1 & 4 & 4 & 4 & 3 & 6 & 3 & 6 & 3 & \begin{tabular}{l|l}
5 & 2 \\
\end{tabular} \\
\hline & & & & & 2 & 4 & 3 & 5 & 2 & 6 & 2 & 4 & 2 & 4 & \begin{tabular}{l|l}
3 & 2 \\
\end{tabular} \\
\hline & & & & & 4 & 4 & 6 & 2 & 5 & 3 & 6 & 3 & 6 & 3 & \\
\hline & & & & 4 & 3 & 6 & 3 & 4 & 3 & 4 & 2 & 4 & 2 & 4 & \begin{tabular}{l|l}
2 & 5
\end{tabular} \\
\hline & & & 1 & 4 & 5 & 2 & 4 & 2 & 2 & 3 & 6 & 3 & 6 & 3 & \begin{tabular}{l|l}
5 & 2
\end{tabular} \\
\hline & & & 2 & 4 & 2 & 5 & 3 & 2 & 2 & 4 & 2 & 4 & 2 & 4 & \\
\hline & & 1 & 4 & 3 & 6 & 3 & 4 & 3 & 4 & 3 & 6 & 3 & 6 & 3 & \\
\hline & 1 & 4 & 3 & 6 & 2 & 6 & 2 & 6 & 2 & 6 & 2 & 4 & 3 & 5 & \begin{tabular}{l|l}
2 & 5 \\
\end{tabular} \\
\hline & 1 & 5 & 4 & 3 & 4 & 3 & 4 & 3 & 4 & 3 & 4 & 2 & 2 & \begin{tabular}{|l|l|} 
& \\
\end{tabular} & \begin{tabular}{l|l}
5 \\
\end{tabular} \\
\hline & 2 & 4 & 2 & 6 & 2 & 6 & 2 & 6 & 2 & 6 & 3 & 2 & 2 & 4 & \\
\hline & 4 & $3 \mid$ & 5 & 3 & 4 & 3 & 4 & 3 & 4 & 3 & 5 & 3 & 4 & 3 & \\
\hline & & 4 & & & 3 & 6 & 2 & 5 & 3 & 5 & 2 & 5 & 3 & \begin{tabular}{l|}
6 \\
\end{tabular} & \begin{tabular}{|l|l}
2 & 5 \\
\end{tabular} \\
\hline & & & & & & & & & & & & & & & \\
\hline
\end{tabular}

Fig. 6 Fuel loading pattern for ABWR core simulation

operation using a 3-D core simulator, NEREUS, and compared the major parameters of core performance, such as k-effective, reactivity margin, shutdown margin, maximum linear heat generation rate (MLHGR) and minimum critical power ratio (MCPR). The core design condition used is summarized in Table 2 and the fuel loading pattern is shown in Fig. 6. The fuel loading pattern was a rotational symmetry and the control cell was formed with the 4th cycle high burnup fuels.

Figure 7 shows the comparison of k-effective versus cycle exposure. The k-effective value, calculated using the group constants generated by MCNP-BURN2, is about $0.7 \% \Delta \mathrm{k}$, which is larger than TGBLA.

The difference is most likely caused by the nuclear data library difference between JENDL-3.3 and ENDF-B/V, since the difference was consistent with the differences in which have been reported by some critical experiment analyses. $^{8,9)}$

Figure 8 shows the comparison of excess reactivity $(\% \Delta \mathrm{k})$. The difference was almost constant during the fuel burnup and the value $(\sim 0.7 \% \Delta \mathrm{k})$ was consistent with the difference of the above k-effectives.

The comparison of MLHGR is shown in Fig. 9. The comparison of MCPR, which is very important for the transient analysis, is shown in Fig. 10. The difference of MLHGRs was less than 3\%. The MLHGR calculated using the group constants generated by MCNP-BURN2 was smaller than that calculated using the group constants generated by TGBLA. The MCPR calculated using the group

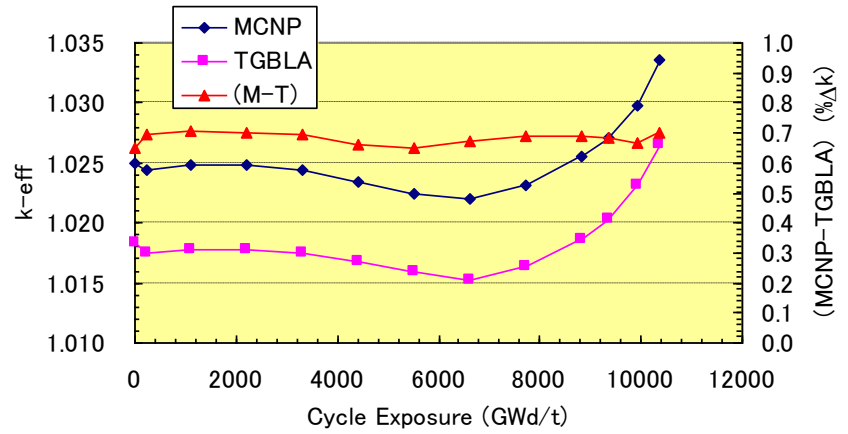

Fig. 7 Comparison of k-effective

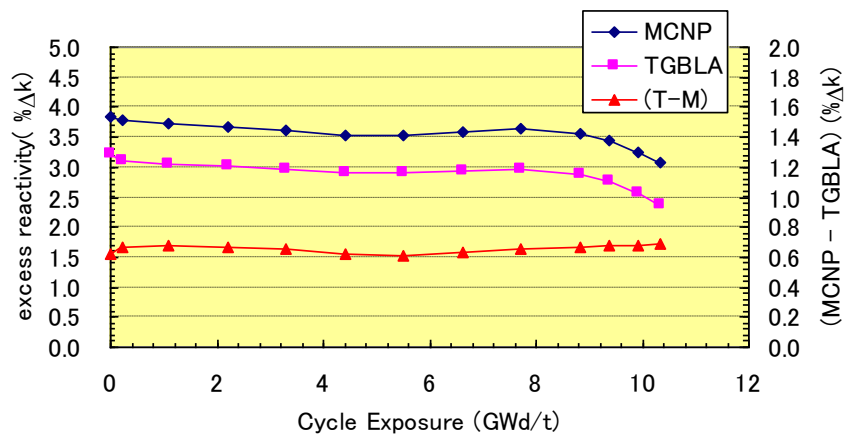

Fig. 8 Comparison of excess reactivity

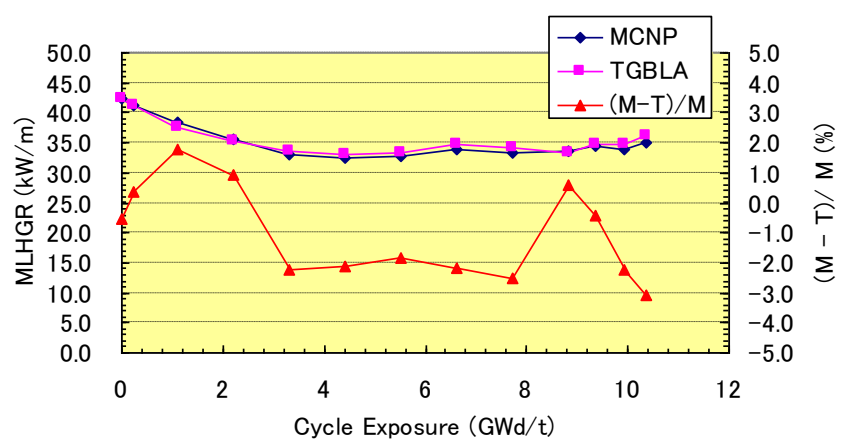

Fig. 9 Comparison of MLHGR

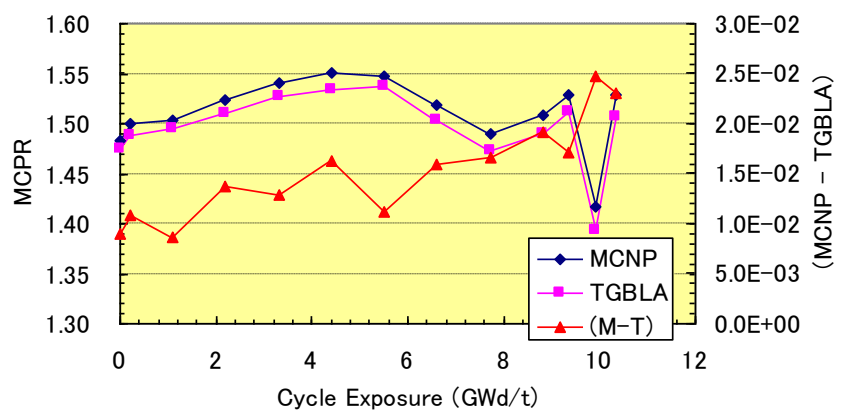

Fig. 10 Comparison of MCPR

constants generated by MCNP-BURN2 was about 0.006 to 0.03 higher than that calculated using the group constants generated by TGBLA. These results show that both calculations are consistent with each other.

We studied the effects of statistical deviations on multi-group constants. We calculated the sensitivity of multi-group constants by changing the number of neutron histories. As an example, Fig. 11(a) shows the change of absorption cross-section as a result of changing the number 


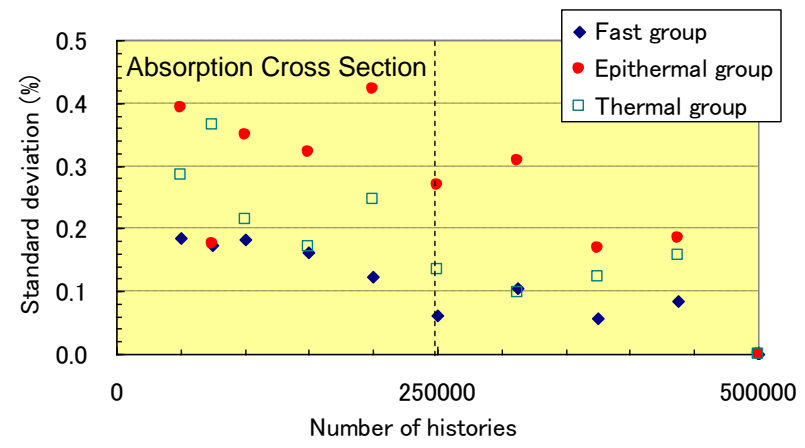

Fig. 11 (a) Change of standard deviation of absorption cross-section vs. number of neutron histories

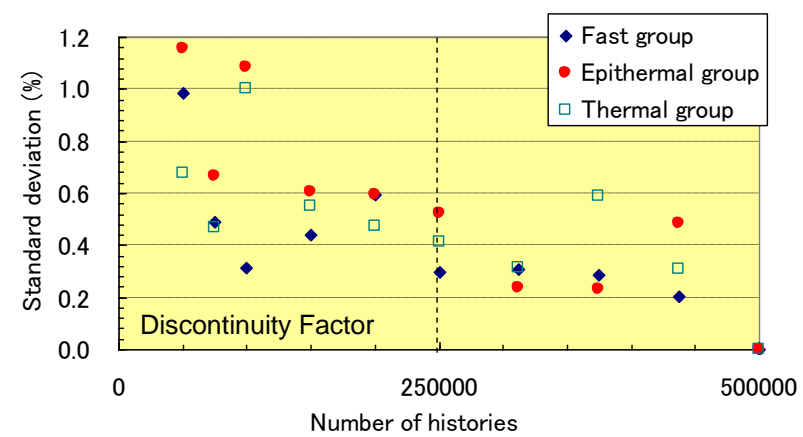

Fig. 11 (b) Change of standard deviation of discontinuity factor vs. number of neutron histories

of histories. Figure 11(b) shows the change in the discontinuity factor as a result of changing the number of histories. The result of 500,000 histories case calculation was used as the standard case. The statistical error of neutron flux in a fuel region was less than $0.5 \%$. The statistical error of reaction rate in a fuel region depends on nuclides and the type of reaction. The statistical error of fission rate or absorption rate for major nuclides such as ${ }^{235} \mathrm{U},{ }^{238} \mathrm{U}$ or ${ }^{239} \mathrm{Pu}$ was approximately $1 \%$. As shown in Fig.11, even if the number of neutron histories is reduced by half, the deviations in the absorption cross-section are at most $0.3 \%$. In the same way, the deviations of discontinuity factor are at most $0.5 \%$.

\section{Application to Plant Transient Analysis}

Using the equilibrium ABWR core described in Section III as the initial core condition, we carried out some typical plant transient analyses using a best-estimate BWR safety and transient analysis code, TRACT. ${ }^{10)}$ TRACT simulates the BWR reactor vessel and core region in 3-D geometry. Other major BWR plant systems, including control systems, are also simulated. The neutron kinetics model used in TRACT is consistent with that in NEREUS at its initial point and it is solved with a modified quasi-static method. ${ }^{11)}$

Figure 12 shows the comparison of the reactor power responses after all recirculation pumps has been tripped. In this case, we used the core condition with the cycle burnup of $9.9 \mathrm{GWd} / \mathrm{t}$ when the MCPR difference is largest between the MCNP core, created with the three group constants by MCNP-BURN2, and the TGBLA core, created with the

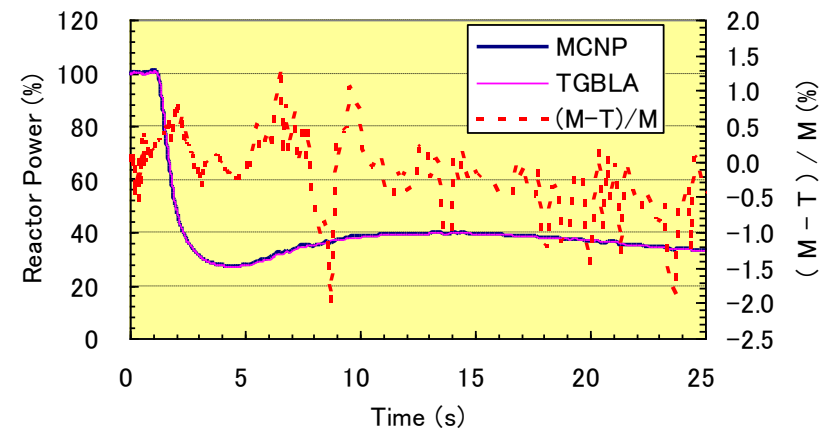

Fig. 12 Comparison of reactor power responses

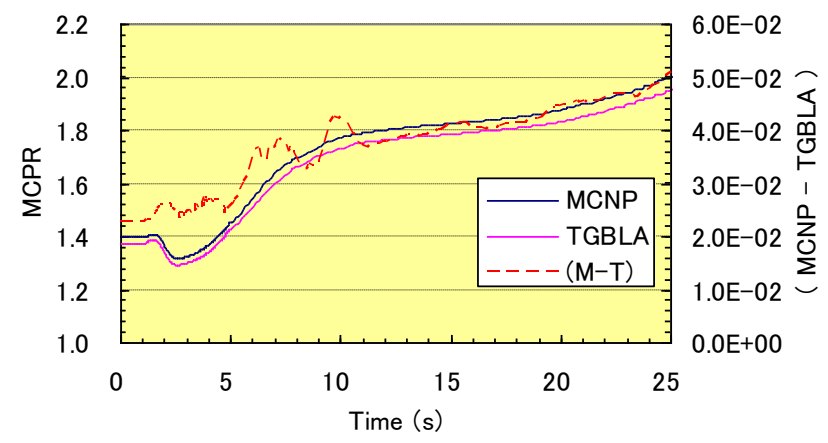

Fig. 13 Comparison of MCPR responses

three group constants by TGBLA. The relative difference of the reactor power responses is less than $2 \%$.

Figure 13 shows the comparison of the MCPR responses. The MCPR responses in this figure are calculated by TRACT, and therefore the initial MCPR values are slightly larger than those by NEREUS, about 0.02 . The difference between MCPRs increases over time. However, the difference at the minimum value of MCPR is almost the same as the initial difference, about 0.025 . The MCPR value using the MCNP core is larger than that using the TGBLA core.

Finally, we will give one example of application of a pin-power reconstruction model for a transient analysis. The fuel rod integrity as a result of the heat removal by the core coolant during the transient event is very important for the plant transient performance evaluation. MCPR is commonly used as the safety index for the fuel integrity evaluation during plant transient events. The fuel pin-power distribution, especially the location of highest pin-power is very important for the MCPR evaluation. Thus, we applied a pin-power reconstruction model to plant transient analyses to evaluate the sensitivity of the pin-power change to the transient responses of the MCPRs.

We chose a core power oscillation induced by neutron-thermal hydraulic coupled instability to be the transient phenomenon. Figure 14 shows the STEP-IIIA assembly configuration used for the ABWR equilibrium core calculation and the fuel pin position, the power of which is analyzed below. It is a $9 \times 9$ lattice fuel assembly and it contains 8 part length rods in order to reduce the core pressure drop to improve the channel stability, the mechanism of which is based on the density-wave oscillation. 


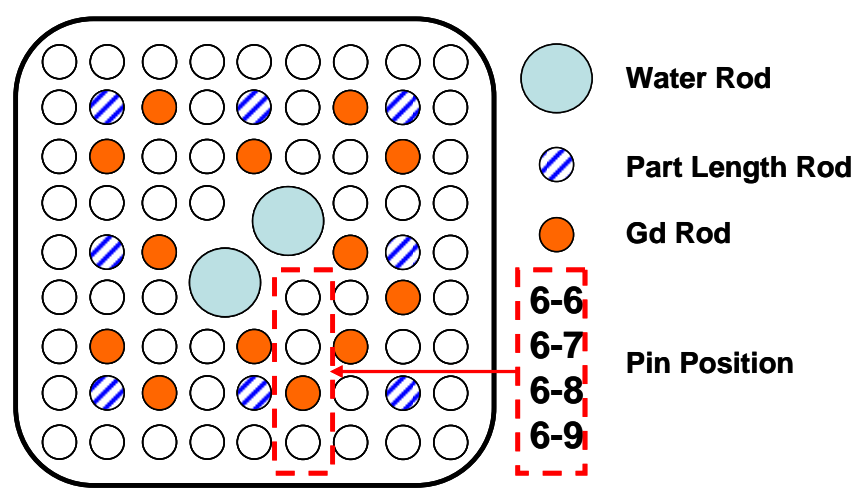

Fig. 14 Fuel rod configuration and pin position in STEP-IIIA type fuel assembly

Figure 15 shows the hot channel's (with the largest radial power peak in a core) power response compared to the fuel pin power located at the axial exit region of the hot channel. All power was normalized at each initial value. The amplitude of the channel power reached about $50 \%$, however the largest pin power amplitude was less than $4 \%$. The channel power responses were in-phase with the center positioned pin power and out-phase with the peripherally positioned pin power at the axially core exit region. The pin power amplitude of the gadolinia bearing rod at the position of 6-8 was quite small. The MCPR responses reflected with the pin power change had almost the same value as the response calculated without the pin power change. The difference was only 0.003 when the channel power amplitude reached $50 \%$. So the effect of the pin power change was negligible during such a neutron flux oscillation phenomenon. However, the situation might be changed in the case of a plant transient event with a control rod movement during which the pin power could change drastically.

\section{Conclusion}

We developed a multi-group constants generation system for a 3-D core simulator using the continuous energy Monte Carlo technique. Comparing the developed system to the current system based on a deterministic lattice code, the generated multi-group constants were in agreement with each other.

We applied the system to an ABWR equilibrium core analysis. Major parameters for core performance such as k-effective and MCPR were in agreement with the current system, within the difference allowed by the nuclear library.

We also applied the equilibrium core to typical plant transient analyses. The major transient related parameters were consistent with those in the current system.

Finally, we confirmed the validity of the continuous energy Monte Carlo based multi-group constants generation system, the core simulation, and the plant transient calculation. We will apply the developed system to research and

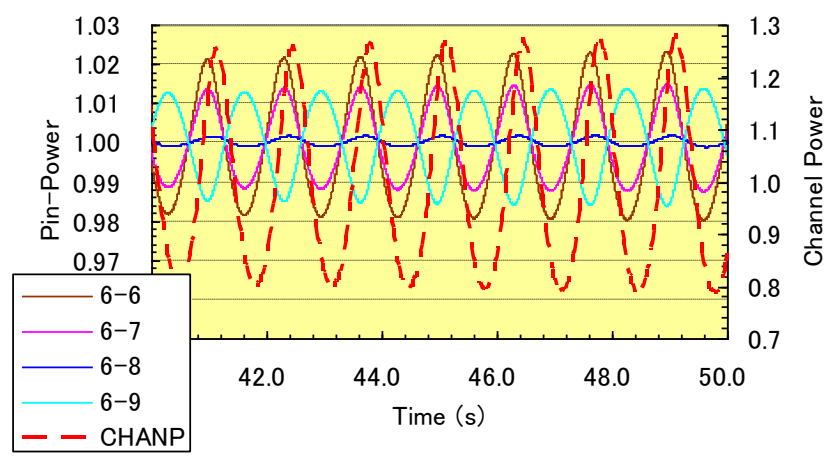

Fig. 15 Comparison of pin power and channel power responses

design next generation nuclear fuels, cores, and plants.

\section{References}

1) Y. Ando, K. Yoshioka, I. Mitsuhashi, K. Sakurada, S. Sakurai, "Development and Verification of Monte Carlo Burnup Calculation System," Proc. of 7th Int. Conf. on Nuclear Criticality Safety (ICNC2003), Tokai, Japan, Oct 20-24, 2003, JAERI-Conf 2003-019, 494-499 (2003).

2) K. Okumura, T. Mori, M. Nakagawa, K. Kaneko, "Validation of a Continuous-Energy Monte Carlo Burn-up Code MVP-BURN and Its Application to Analysis of Post Irradiation Experiment," J. Nucl. Sci. Technol., 37, 128-138 (2000).

3) M. Tohjoh, M. Watanabe, A. Yamamoto, "Application of continuous-energy Monte Carlo code as a cross-section generator of BWR core calculation," Ann. Nucl. Energy, 32, 857-875 (2005).

4) K. Yoshioka, Y. Ando, "Multi-group Scattering Matrix Generation Method Using Weight-to-Flux Ratio Based on a Continuous Energy Monte Carlo Technique", J. Nucl. Sci. Technol., 47, 908-916(2010).

5) T. Iwamoto, M. Yamamoto, "Advanced Nodal Methods of the Few-Group BWR Core Simulator NEREUS”, J. Nucl. Sci. Technol., 36, 996 -1008(1999).

6) M. Yamamoto et al., "New Physics Models Recently Incorporated in TGBLA", Proc. Of Int. Top. Mtg. on Advances in Mathematics, Computation and Reactor Physics, Pittsburgh, U.S.A (1991).

7) J. F. Briesmeister, (Ed.), MCNP - A General Monte Carlo $N$-Particle Transport Code, Version 4A, LA-12625, Los Alamos National Laboratory (LANL) (1993).

8) Database for the International Criticality Safety Benchmark Evaluation Project, http://icsbep.inel.gov/dice.shtml

9) K. Shibata et al.," Japanese Evaluated Nuclear Data Library Version 3 Revision-3: JENDL-3.3," J. Nucl. Sci. Technol., 39, 1125 -1136 (2002).

10) T. Fukunaga et al.," BWR Transient Analysis Validation with TRACT Code," Proc. NUTHOS-8, Oct. 10-14, 2010, Shanghai, China (2010), [CD-ROM].

11) T. M. Sutton, B. N. Aviles, "Diffusion Theory Methods for Spatial Kinetics Calculations," Prog. Nucl. Energy, 30, 119-182 (1996). 